REDES- Revista hispana para el análisis de redes sociales.

Vol.3,\#6, sept-nov. 2002.

http://revista-redes. rediris.es

\title{
Amistad e identificación: las micro fundaciones de las pertenencias macro. Amigos europeos e identidad europea $^{1}$
}

\author{
Ainhoa de Federico de la Rúa ${ }^{2}$ \\ CLERSÉ-IFRESI, Universidad de Lille (Francia) \\ Universidad Pública de Navarra (España)
}

\begin{abstract}
Macro belongings to abstract "imagined communities" (Anderson 1983), such as nations, are grounded on the generalized metonymy transfer, encouraged by institutions, to the inhabitants of a certain territory, of the interpersonal experience of trust and solidarity.

Indeed, trust and solidarity are first learned in the interactions taking place sphere of kinship. Later on, it is through the learning of solidarity in friendship by age groups that individuals are able to go beyond primary family solidarity into more abstract solidarity in universalistic societies as ours (Eisenstadt 1956).

The national social contract is ideologically founded on the ideal model of friendship solidarity. The most explicit example of it being the French motto "liberté égalité fraternité". This doesn't mean that there is an exact correspondence between the two kinds of relationships, as Eisenstadt (1984) points out the ideal fraternity announced by the nation serves to hide and justify, the power and instrumentality that the order of the nation-state exerts. At the same time, friendship (in its large conception) sustains the social contract at the same time as it has to be relegated to the private sphere due to its subversive potential (Paine 1969).
\end{abstract}

Friendship relations often stay within the sphere of nation-states, since their institutions structure interaction and interdependencies by the division of labor. However what happens with cross-national friendships? The paradoxical figure of "foreign friends" introduces a tension between the foundations of abstract solidarity and the experience of interpersonal solidarity. Is this tension relegated to be solved in the private sphere? May it have an impact on the claims of public macro belongings?

Furthermore, in the European context there is an institutional setting allowing and trying to encourage European identity. Can such micro European friendships give a

\footnotetext{
${ }^{1}$ Agradezco a Alexis Ferrand, Narciso Pizarro, Ronald Breiger y Reyes Herrero sus comentarios sobre versiones anteriores.

${ }^{2}$ Enviar correspondencia a: Ainhoa de Federico, federico@univ-lille1.fr
} 
content to the, otherwise empty in terms of emotional attachment, macro legal shell of European citizenship encouraging European identity?

We examine the effects of cross-national friendships in a sample of 218 European exchange students on their perceptions of other Europeans as foreign and opening on their European identity. 


\section{Resumen}

Las pertenencias a "comunidades imaginadas" macro (Anderson 1983), tales como las naciones, están fundadas en la transferencia metonímica generalizada, fomentada por las instituciones, a los habitantes de cierto territorio, de la experiencia interpersonal de confianza y solidaridad.

En efecto, la confianza y la solidaridad son primero aprendidas en las interacciones que tienen lugar en la esfera de la familia. Después, mediante el aprendizaje de la solidaridad a partir de la amistad en grupos de pares de la misma edad, los individuos son capaces de pasar de la solidaridad primaria familiar hacia formas de solidaridad más abstractas en sociedades universalistas como las nuestras (Eisenstadt 1956).

El contrato social nacional está ideológicamente fundado en el modelo ideal de la solidaridad en las relaciones de amistad. El ejemplo más explícito de ello es el lema francés "liberté, égalité, fraternité". Esto no quiere decir que exista una correspondencia exacta entre estos dos tipos de relación, tal como Eisenstadt (1984) señala, la fraternidad ideal anunciada por la nación sirve para ocultar y justificar el poder y la instrumentalidad que el orden de la nación-estado ejerce sobre sus miembros. Al mismo tiempo, la amistad (en su acepción más amplia) sostiene el contrato social al mismo tiempo que debe ser relegada a la esfera privada debido a su potencial subversivo (Paine 1969).

Las relaciones de amistad a menudo ocurren dentro de la esfera de los estadosnación, puesto que sus instituciones estructuran la interacción y las interdependencias entre los actores a partir de la organización de la división social del trabajo. Sin embargo, ¿qué sucede con las relaciones transnacionales? La figura paradójica del "amigo extranjero" introduce una tensión entre los fundamentos de la solidaridad abstracta y la experiencia de la solidaridad interpersonal. ¿Queda esta tensión relegada a ser resuelta en la esfera privada? ¿O acaso tiene una incidencia en las pertenencias públicas macro sociales reclamadas y proclamadas por los actores?

Actualmente, en Europa existe un contexto institucional que permite e intenta promover la identificación con Europa. ¿Podrían tales amistades europeas dar un contenido al envoltorio legal macro de la ciudadanía Europea - por otra parte vacío en términos de apego emocional- fomentando la identidad Europea?

Examinamos el efecto de relaciones de amistad trans-nacionales en una muestra de 218 estudiantes Europeos, en su mayoría participantes en programas Europeos de intercambio, sobre la percepción y categorización mutua en tanto que extranjeros, abriendo la cuestión de la identidad Europea.

\section{I dentidad, nación, amistad}

Este artículo trata sobre "identidad" y sobre "identidad europea" y su relación con procesos interpersonales de establecimiento de relaciones de amistad. El término "identidad" es uno de los más vagos y confusos en sociología, dado que su uso profano tiende a confundirse con la comprensión sociológica de éste. El modo en que la "identidad" nos interesa aquí es tal como se utiliza comúnmente por los profanos para referirse a lo que en términos especializados podría definirse como los sentimientos de pertenencia a una - por lo menos deseada- "comunidad imaginada política, soberana y limitada territorialmente" (Anderson 1986). 
Es posible distinguir varias dimensiones en este tipo de "identidad":

1) Las definiciones (macro) de la ciudadanía y los derechos legales llevadas a cabo y garantizadas por los Estados y otras instituciones legítimas garantes del orden. En Europa, con un lugar progresivamente más prominente, la Unión Europea.

2) Los discursos (macro) sobre la "identidad" ligados a la emergencia histórica de las naciones y el nacionalismo que pretenden conferir a una comunidad imaginada con una supuesta etnicidad común (antepasados, cultura y medios de comunicación) la legitimidad de devenir, en términos de Schnapper (1994), una "comunidad política de destino". ${ }^{3}$

3) Los sentimientos individuales (micro) de pertenencia e identificación a las unidades - eventualmente en competición, tensión o conflicto- definidas por las instituciones (1) y/o por los discursos ideológicos (2).

La relación entre estas tres dimensiones es compleja y representa el objeto de largos y extensos debates teóricos e ideológicos en las ciencias sociales, en particular la relación entre la ciudadanía institucional (demos) y la "nacionidad" (ethnos) (Schnapper 1994, Lamo de Espinosa 1995 entre muchos otros). Sin embargo el estudio de los sentimientos subjetivos individuales de pertenencia a las "comunidades imaginadas" son a menudo, incluso de forma periódica, estudiados empíricamente (Eurobarómetros, encuestas del CIS...) pero poco teorizados ${ }^{4}$.

La atención principal de nuestra investigación trata de las (micro) identificaciones subjetivas tal como pueden ser formadas o influidas por (meso) redes de amistad en el marco de los (macro) discursos ideológicos y los contextos institucionales. El dominio de aplicación es Europa y la "identidad Europea".

\footnotetext{
${ }^{3}$ Se podría decir que la primera dimensión, la definición institucional de la ciudadanía, resulta de una reificación de la segunda dimensión, los discursos sobre la identidad, mediante la institucionalización y operacionalización de los consensos logrados por las distintas élites, en tensión, portadoras de los discursos a los órganos de representación política.

${ }^{4}$ Más prometedora parece la investigación europea EURONAT, en curso, dirigida por Bo Strath y Anna Triandafyllidou (Instituto Universitario Europeo de Florencia) reuniendo equipos interdisciplinares de toda Europa.
} 


\section{Figura 1. El caso de la identidad europea: una aplicación del "barco" de Coleman (1992).}

\section{Nivel macro}

2) Discursos ideológicos

1) Ciudadanía institucional

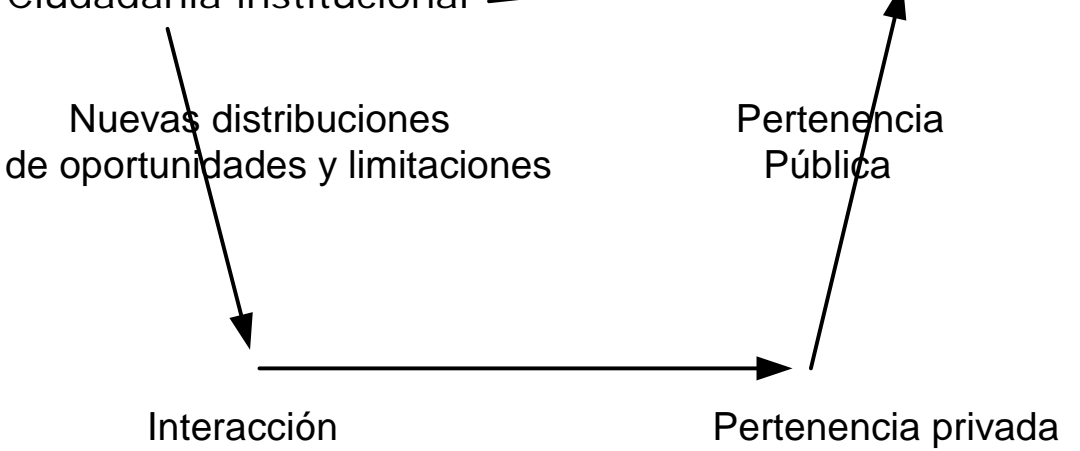

Identidad europea

3) Procesos de amistad: selección, influencia, regulación diádica y triádica, establecimiento de igualdad, confianza y solidaridad.

\section{Nivel micro}

1) Las definiciones institucionales de la ciudadanía pueden tener un impacto directo sobre los sentimientos de pertenencia a cierta unidad (comunidad imaginada): el hecho de que exista una categoría administrativa de pertenencia y los derechos asociados a ella puede causar que los individuos se sientan identificados con ésta. Sin embargo, esto parece bastante abstracto y queda lejos de los elementos más afectivos y simbólicos presentes en las identificaciones a comunidades imaginadas. A pesar de ello, no olvidemos que, la existencia de estas definiciones de la ciudadanía a) legitiman los sentimientos de pertenencia, puesto que corresponden con la categoría "oficial", b) producen la organización institucional que estructuran las interacciones y las experiencias de los individuos, lo que a su vez puede tener un impacto sobre las oportunidades de interacción y los procesos de identificación ${ }^{5}$.

La convergencia institucional de las sociedades Europeas (Mendras 1997), así como la construcción de la Unión Europea y la definición de ciertas esferas de ciudadanía Europea y derechos de los Europeos, ofrecen la matriz institucional que podría acoger los sentimientos de pertenencia e identificación. Además, éstos permiten

\footnotetext{
5 No olvidemos el importante papel de instituciones impuestas por los estados como la escuela obligatoria para todos los ciudadanos, y el alistamiento masculino obligatorio, en la construcción de las naciones modernas.
} 
estilos de vida y trayectorias biográficas genuinamente "Europeas" ${ }^{6}$ para aquellos individuos con la capacidad y/o el capital económico, humano, social, de disfrutar de sus derechos Europeos.

2) Los discursos ideológicos sobre la "identidad nacional" basados en una supuesta etnicidad común (antepasados y cultura) y la existencia de medios comunes de comunicación, han tenido, por otra parte, bastante éxito -aunque nunca completamente- en formar y movilizar las identificaciones y los sentimientos de pertenencia de los individuos. Existen pocas pruebas de la existencia de un discurso sobre la "identidad Europea" ampliamente aceptado compitiendo con los nacionales, y por el contrario, mucha discrepancia sobre los términos en que éste debería ser definido. Los textos oficiales de la Unión Europea han adoptado, siguiendo parcialmente el modelo de las identidades nacionales, una definición etnoculturalista y liberal (Hansen 2000). Los científicos sociales (Habermas 1999, Touraine 1999, Therborn 1999, Soysal 2001...) a menudo proponen definiciones con un mayor énfasis en la ciudadanía, los valores universales y los derechos humanos que sobre rasgos culturales particulares. Sin embargo, entre la población, tal como informan los Eurobarómetros, la identidad europea aparece con mucha frecuencia entre las identidades avanzadas por los encuestados, a menudo en segundo lugar tras la identidad nacional. Los Eurobarómetros sólo proponen un posicionamiento combinando identidades nacional y europea, pero recordemos que la identidad nacional no siempre es la primordial, a menudo (según los países) aparece tras niveles de identificación más locales como la ciudad o la región.

Los Eurobarómetros también nos enseñan que aquellos individuos más proclives a sentirse europeos (lo mismo que los más propensos a sentirse "nacionales") son aquellos con más estudios, con profesiones de categorías sociales elevadas, mayores ingresos, urbanos, y entre las generaciones más jóvenes. Es razonable emitir la hipótesis de una correspondencia entre el poder, pero de manera más general, las oportunidades ofrecidas por el nivel de identificación, y la propia identificación.

3) Entre las oportunidades que abre la definición de los derechos ciudadanos, en este caso los derechos europeos - y aquí descendemos al nivel micro- está el de mayor interacción con otros individuos europeos, y eventualmente el

\footnotetext{
${ }^{6}$ No sería la primera vez que esto se produce en la historia, por no volver a ejemplos evidentes como el de la Respublica Litteraria de la Europa renacentista (Pomian 1990, p91-102), más recientemente en el siglo $\mathrm{XIX}$ y a principios del $\mathrm{XX}$, antes de la II guerra mundial y el auge de los nacionalismos, los intelectuales y la academia científica eran eminentemente europeos y las universidades europeas contaban con mayores números de extranjeros entre sus filas que hoy en día (Manitakis 2000).
} 
establecimiento de todo tipo de relaciones, entre ellas las de amistad, es decir, relaciones de confianza y solidaridad. ¿Por qué centrarse en un tipo de relación tan privada y aparentemente insignificante como la amistad, cuando anteriormente hemos mencionado elementos sociales "serios" como las instituciones y los discursos ideológicos? La elección de la amistad como la relación estudiada no es fortuita: la ideología de la nación está fundada de manera explícita y funcional en los modelos ideales de confianza y solidaridad de las relaciones de amistad y parentesco lateral. Para ilustrar esto con un ejemplo claro, uno de los primeros estados nación históricos, Francia, se dio como lema liberté égalité fraternité. Por otro lado las alusiones a la "madre patria" que convierten a los conciudadanos en “hermanos ideales" son típicas en los discursos nacionalistas. De hecho en la literatura sobre la nación encontramos como colofón de la definición de Anderson que:

"es imaginada en tanto que comunidad, porque a pesar de la desigualdad y la explotación que pueda predominar en cada una, la nación siempre es concebida como una profunda camaradería horizontal. Finalmente es esta fraternidad la que la ha hecho posible en los últimos dos siglos" 7 .

De manera similar, en las aproximaciones que sitúan la amistad en el interior de la matriz de las instituciones macro sociales encontramos Eisenstadt y Roninger (1984) afirmando que, gracias a la existencia de expresiones concretas de confianza y solidaridad en las relaciones de amistad y parentesco y mediante su relegación a la esfera privada, el estado (nacional) puede proclamar estos valores ideales al mismo tiempo que ejerce relaciones instrumentales y de poder ${ }^{8}$. Es decir, que los ideales nacionales y la cohesión nacional se sostienen por la experiencia cotidiana interpersonal de solidaridad y confianza en las comunidades personales de amigos y parientes. Para ilustrar esto tomemos como punto de partida a Fichte (1955:3) cuando se dirige a la nación alemana:

"Estoy hablando a todos los alemanes, sobre todos los alemanes, sin excepción, desdeñando a propósito todas las diferencias resultado de eventos profanos por los que nuestra nación ha sufrido desde hace siglos [Ich rede fuer Deutsche schlechtweg, von Deutschen schlechtweg, nicht anerkennend, sondern durchaus beiseitesetzend undwegwerfend all die trennenden

\footnotetext{
${ }^{7}$ La traducción y la itálica son mías (Anderson, 1984, p7).

${ }^{8}$ Para encontrar una exposición más detallada sobre la relación entre amistad y nación ver de Federico 2001 http://www.mmu.ac.uk/gsa/first_conference.html.
} 
Unterscheidungen, welche unselige Ereignisse seit Jahrhunderten in der einen Nation gemacht haben]"

Según el idealismo de Fichte, existe una esencia de "lo alemán" y por ello un alemán sería igual a otro alemán en raza, clase, cultura etc. Es evidente que tal alemán no existe, mucho menos de forma generalizada. Sin embargo podríamos argüir que los individuos pueden experimentar esta igualdad en la práctica en el caso ideal -también hallado empíricamente- que ofrece el modelo del "amigoedad", el "amigo verdadero", definido por Ferrand (1993,p. XLIII-XLVII). LoS "amigos-edad" son individuos en calidad paradójica de "hermanos" fuera del parentesco, cuyos lazos son irrevocables, a vida y a muerte, y son más iguales incluso que los hermanos verdaderos (con la excepción mitológicamente valorada de los hermanos gemelos), ya que no existe entre ellos siquiera distinción de rango y a menudo tampoco de otras características sociales. Los amigos de verdad, los "amigos-edad" son los "iguales" sociales por excelencia.

Incluso sin hacer referencia a teorías macro de la amistad que la sitúen en la matriz institucional de la sociedad en relación con las otras instituciones reguladoras "duras" de la sociedad, y que son las que permitirían la transición de micro a macro, encontramos argumentos para defender la "línea micro": Además de ser iguales morales, los amigos tienden a ser socialmente similares y a devenir parecidos como consecuencia de su relación de amistad.

\section{¿Amigos extranjeros?}

Es probable que, por la mera existencia de un estado (nacional) organizando la vida social, la amistad y el parentesco tiendan a tener lugar dentro de sus límites. En este caso el paralelismo entre la comunidad personal privada que produce la confianza y la solidaridad concretas y la comunidad imaginada pública que obtiene crédito gracias a la anterior son coherentes. ¿Pero qué sucede cuando esta correspondencia no es tal? ¿Qué ocurre cuando la comunidad personal está compuesta por ciudadanos de diferentes naciones-estado? Dado el marco de la construcción europea y los derechos a la movilidad y al trabajo que ésta permite para los ciudadanos de la UE en su espacio, es probable que esto suceda cada vez más, al menos en el caso de los ciudadanos con mayor formación.

La hipótesis que adelantamos aquí es que, dado que los sentimientos de pertenencia e identificación a comunidades imaginadas públicas, es decir la solidaridad con esas comunidades abstractas, está basada en el modelo ideal de la 
amistad fraternal y apoyado en la experiencia concreta de confianza y solidaridad a través de la amistad y el parentesco con miembros de estas unidades, transferidas de forma metonímica al conjunto de la unidad. El desarrollo significativo de confianza y solidaridad con miembros de otras unidades puede tener un impacto en la identificación y sentimientos de pertenencia: aumentando su ámbito, haciéndolas relativas respecto a otras o compartidas con identificaciones a otras unidades. ${ }^{9}$

Sin embargo, dado que la amistad se ve relegada a la esfera privada, es necesario realizar una distinción entre dos niveles de impacto potencial:

1) Primero, el establecimiento de relaciones de amistad puede cambiar las percepciones de "quien pertenece y quien no pertenece". Puesto que la pertenencia y solidaridad con una comunidad imaginada es mediada primero por la pertenencia y solidaridad hacia comunidades personales concretas, la inclusión en una misma comunidad personal podría conllevar el sentimiento o la percepción de pertenecer a la misma comunidad abstracta, incluso si esta no está especificada de manera explícita. La justificación de cual debiera ser la comunidad imaginada "receptora" puede aparecer a posteriori. Por lo tanto, por el propio proceso en que dos extraños devienen amigos, personas (oficialmente) extranjeras la una a la otra pueden terminar no percibiéndose como tales. Los contextos institucionales pueden sin duda favorecer estas percepciones. Hasta aquí estamos en el nivel privado de transformación de las percepciones y la caracterización mutua como consecuencia del establecimiento de una relación de amistad.

2) El ámbito de influencia de la amistad puede sin embargo trascender la esfera privada. La existencia de relaciones trans-nacionales, especialmente si éstas son fuertes tanto en términos diádicos (duración, intensidad subjetiva, calidad de los contenidos...) o en términos estructurales (centralidad, imbricación) y las características generales de la red personal (densidad, composición...), pueden favorecer la emergencia o aumentar la importancia relativa de los sentimientos de pertenencia e identificación con comunidades imaginadas capaces de incluir a los miembros de las comunidades personales.

Por el momento tan solo tenemos resultados empíricos sobre los efectos posibles de la amistad en la esfera privada. Los análisis que presentamos aquí se refieren a este tipo de influencia a partir del caso que estudiamos.

\footnotetext{
9 Sin duda, el mecanismo o proceso que describimos aquí no es el único que puede fomentar sentimientos de identificación con una comunidad imaginada macro, en nuestro caso "Europa". Todos los elementos simbólicos introducidos en la vida cotidiana por las instituciones, como por ejemplo la llegada del Euro en enero del 2002, contribuyen y aceleran estos procesos.
} 


\section{El caso de estudio: la identidad Europea de estudiantes en programas europeos de intercambio}

La UE es consciente, ya lo era el Consejo de Europa desde los años 50, de que la simple integración económica e institucional no es suficiente para su legitimidad pública y para la unidad europea. Para ello, es necesaria además la emergencia de la conciencia o identidad europea, al menos en cierta parte de la población. En línea con esta reflexión, desde mediados de los años 80 , la UE ha invertido grandes cantidades, primero de ecus, y luego de euros ${ }^{10}$ para fomentar la movilidad estudiante con una doble idea enfatizada explícitamente en su discurso oficial: 1) Los estudiantes europeos en intercambio, entendidos como vectores de cultura, la experiencia intercultural que provocan y en que participan, pueden ser un poderoso catalizador de la formación de la conciencia o identidad europea. 2) La movilidad estudiante es el mecanismo para el desarrollo de una mano de obra móvil y bien altamente educada que perciba la UE como su mercado de trabajo potencial, una idea que parece como la condición sine qua non para la realización del mercado único ${ }^{11}$. Desde su implantación en 1987, tan sólo gracias al programa Erasmus (existen otros muchos) se estima que aproximadamente 1,5 millones de estudiantes universitarios han disfrutado de una estancia de 3 a 12 meses en otro país europeo ${ }^{12}$.

Para nuestra investigación hemos escogido estudiar una muestra de estos estudiantes Erasmus. Esta población, temporalmente móvil, tiene grandes oportunidades de interacción y establecimiento de relaciones de amistad con otros ciudadanos europeos. Además, dado que ésta población se encuentra concentrada en instituciones universitarias, es posible observar tanto los procesos de formación de amistad como sus efectos.

Un cierto número de estudios y evaluaciones, la mayoría financiados por la UE, han sido llevados a cabo a propósito de las experiencias de los estudiantes participando en estos programas por equipos en Kassel (Alemania) y Lille (Francia). Algunos de ellos mencionan la gran importancia concedida por los propios estudiantes a sus nuevas relaciones de amistad y que las relaciones transnacionales son frecuentes (Martineau 1995). Pero ninguno las estudia en profundidad de manera sistemática,

\footnotetext{
${ }^{10}$ Por ejemplo, 840 Millones de euros sólo para el periodo 1990-1995.

${ }^{11}$ Sobre la importancia concedida por la UE a los programas de intercambio de estudiantes europeos y la evolución de su discurso a propósito de la importancia del desarrollo de la conciencia europea mediante éstos ver Ruiz-Gelices et al. (2000).

12 Cifra importante si se compara con los 5 millones estimados de europeos que viven en otro estado miembro de la EU. A estas migraciones también se podrían añadir otros tipos de migraciones temporales: turismo, residencias secundarias, jubilaciones...
} 
mucho menos su impacto sobre la identidad europea de los estudiantes participantes. De hecho, al no relacionar la calidad de las redes personales de los estudiantes con el tipo de identidad europea emergente (o no), los resultados de estas encuestas son a menudo insípidos y decepcionantes ${ }^{13}$.

La presente investigación se basa en datos sobre 218 estudiantes universitarios de la Universidad de Lille (Francia), la Universidad Pública de Navarra (España) y la Universidad de Groningen (Holanda). Entre los 218 consta una muestra de control de estudiantes Españoles de la Universidad Pública de Navarra que no participaron en ningún programa de intercambio. Procedimos mediante un cuestionario estandarizado (en francés, español e inglés) incluyendo varios módulos. Primero un módulo de análisis de redes sociales mediante el que se interrogó a los estudiantes por sus amigos y otras relaciones importantes, tanto el año anterior al intercambio, como durante el intercambio. Este módulo permite examinar las relaciones de amistad (intensidad, contenido, roles...) así como las redes personales de los estudiantes encuestados (densidad, subgrupos, composición...). Un segundo módulo incluía preguntas sobre las identificaciones tanto con comunidades imaginadas definidas territorialmente (localidades, regiones, naciones, Europa, otros) como con otros tipos de comunidades (religiosas, lingüísticas, étnicas...).

Como anunciamos anteriormente presentamos resultados parciales sobre estos aspectos. Primero una breve descripción sobre las relaciones creadas por los estudiantes: con quienes devienen amigos y la presencia de relaciones transnacionales en sus redes personales. Una vez establecida la existencia de relaciones transnacionales, examinaremos los efectos de éstas al nivel de la esfera privada, es decir, el impacto de la amistad en la categorización mutua en tanto que extranjeros. El siguiente paso sería evaluar si las relaciones de amistad son capaces de abolir la "extranjería" sólo en la esfera privada o si estas relaciones (o su importancia, o su acumulación, o su heterogeneidad en las redes personales) tienen un impacto en las esferas públicas de identificación. Esto es, si influyen en las identidades proclamadas por los individuos, modificando las estructuras cognitivas y afectivas de identificación y pertenencia, tal vez otorgando mayor importancia a identidades transnacionales capaces de incluir a los miembros de las comunidades personales. Este segundo paso queda fuera del presente artículo.

\footnotetext{
${ }^{13}$ Más prometedora resulta la propuesta etnográfica de la tesis en curso de Enric Ruiz Gelices.
} 


\section{Descripción de las relaciones}

Cuando examinamos los lazos de amistad establecidos por los estudiantes de esta muestra, vemos que los 218 estudiantes - control incluido- mencionaron 6592 relaciones. El conjunto de estudiantes mencionaron una media de 15 relaciones en cualquier momento. Los estudiantes Erasmus mencionaron 10 relaciones de promedio durante su periodo de intercambio. Respecto al tipo de amigos, nos damos cuenta de que durante el periodo Erasmus el $62 \%$ de las relaciones establecidas son con personas de igual nacionalidad y el $38 \%$ son amistades transnacionales. La población que hemos escogido estudiar crea por lo tanto amistades transnacionales en número considerable, lo que permite estudiar los efectos de éstas. A continuación, el primer paso es examinar cómo se resuelve la tensión introducida por la figura del "amigo extranjero". ¿Podrá la instauración de una relación de amistad entre dos personas de diferentes nacionalidades hacer que la importancia de la nacionalidad se atenúe? ¿Seguirán los amigos considerándose extranjeros mutuos? Y si no es así, ¿fomentará su relación de amistad otras formas de identificación, o será esta relación de solidaridad relegada a la esfera privada? Por el momento podemos contestar a las dos primeras preguntas, relativas al nivel privado.

Antes de examinar los resultados empíricos recordemos las dos lógicas principales implicadas:

1) La lógica de la nación implicaría que la solidaridad se establezca preferentemente entre miembros de la misma nacionalidad y no con miembros externos a ella (ya hemos visto que aunque la tendencia está presente, se dan numerosos casos en que eso no es así). También, de acuerdo con la lógica de la nación las personas de misma nacionalidad no deberían ser consideradas como extranjeras y las personas de nacionalidad distinta deberían ser consideradas como extranjeras.

2) La lógica de la amistad, por otra parte, implicaría que, una vez la amistad establecida, un amigo se convierte en un igual y sus otras características sociales pierden importancia ante esta igualdad. La lógica de la amistad, implicaría que los amigos ya no sean considerados como extranjeros, incluso a pesar de que su nacionalidad diferente pueda ser percibida en algunos casos entre otras características sociales. 
De algún modo, la lógica de la amistad "deformaría" la lógica institucional de la nación al encontrarse en su fundamento. La noción de "extranjería" en este caso, no haría tanto referencia a algo sustantivo o determinado institucionalmente, sino relativo a la pertenencia a un grupo social común, ya sea este una comunidad personal, una comunidad imaginada o ambos mediante el establecimiento de una relación metonímica entre ellos.

Examinemos los datos y veamos qué sucede. Para valorar la posición percibida entre los amigos en esta cuestión de la "extranjería" se preguntó a los estudiantes si consideraban a cada amigo como un extranjero o no y si notaban o no el hecho de que el amigo tenía una nacionalidad diferente. En las respuestas que se propusieron se pretendía capturar el eventual efecto de "deformación" de la amistad.

La pregunta y las respuestas propuestas eran las siguientes:

"Q13. Consideras a estas personas como extranjeras?".

0 . No la considero como extranjera, tiene la misma nacionalidad que yo.

1. No la considero como extranjera aunque me doy cuenta de que tiene una nacionalidad diferente.

2. No la considero como extranjera; y no noto que tiene una nacionalidad diferente.

3. La considero extranjera y me doy cuenta de que tiene una nacionalidad diferente."

La primera posibilidad y la última son evidentes y corresponden a la lógica de la nación: una persona con igual nacionalidad no es extranjera (0); por otra parte una persona con nacionalidad diferente es extranjera (3). Las dos posibilidades intermedias corresponden a los efectos que se podrían esperar de la lógica de la amistad en la percepción de amigos trans-nacionales. Introducimos un matiz entre notar la nacionalidad diferente como una característica social (1) y dejar de percibirla (2). En este caso la "extranjería" es completamente eliminada de la esfera de la amistad. Esperamos una progresión en el nivel de intimidad o fuerza de la relación y la percepción del amigo como cada vez menos extranjero. 
Tabla 1. Amigos transnacionales y percepción de extranjería. *

\begin{tabular}{|l|c|c|c|}
\hline Percepción de la extranjería & Número & Porcentaje & $\begin{array}{c}\text { Amigos } \\
\text { Extranjeros (\%) }\end{array}$ \\
\hline $\begin{array}{l}\text { I gual nacionalidad, no } \\
\text { considerado extranjero }\end{array}$ & 1337 & $62 \%$ & --- \\
\hline $\begin{array}{l}\text { No considerado extranjero, } \\
\text { nacionalidad percibida. }\end{array}$ & 500 & $23 \%$ & $\mathbf{6 0 \%}$ \\
\hline $\begin{array}{l}\text { No considerado extranjero, } \\
\text { nacionalidad olvidada }\end{array}$ & 206 & $9 \%$ & $\mathbf{2 5 \%}$ \\
\hline Extranjero & 130 & $6 \%$ & $\mathbf{1 5 \%}$ \\
\hline Totales & $\mathbf{2 1 7 3}$ & $\mathbf{1 0 0 \%}$ & $\mathbf{1 0 0 \%}$ \\
\hline
\end{tabular}

(*)Relaciones establecidas sólo por Erasmus y durante su periodo en el extranjero.

Tal como observamos la amistad opera una deformación de las fronteras percibidas de extranjería. Las personas de igual nacionalidad representan el $62 \%$ y la lógica de la nación supone que no sean consideradas extranjeras ${ }^{14}$. Sin embargo, del restante $38 \%$, solo $6 \%$ de los amigos son considerados como extranjeros.

Si excluimos los amigos de igual nacionalidad, observamos que el $85 \%$ de los amigos transnacionales no son considerados como extranjeros, sólo el $15 \%$ son considerados extranjeros. La amistad ejerce claramente un movimiento de las fronteras de extranjería percibida en la gran mayoría de los casos. Si examinamos el tipo de percepción que tienen los estudiantes sobre sus amigos transnacionales, observamos que en la mayoría de los lazos (el 60\%) los amigos no son considerados extranjeros, aunque su nacionalidad distinta sí es percibida. ¿Quiere esto decir que aquellos que responden de este modo consideran otras unidades de pertenencia a la que ellos y sus amigos pertenecen, y respecto a la que no son extranjeros mutuos? Otras relaciones de amistad transnacional (el 25\%) implican que el amigo no es percibido como extranjero y también que su nacionalidad no es siquiera percibida. ¿Quiere esto decir que la relación de amistad ha alcanzado un nivel de intimidad tal que toda otra categorización social, incluida la nacionalidad, considerada tan primordial por algunos, pierde importancia? Finalmente, en el $15 \%$ de los casos, la minoría, el establecimiento de una amistad no implica un cambio de percepciones sobre la extranjería del amigo.

\footnotetext{
${ }^{14}$ Hubiéramos podido examinar en más detalle si eso sucede. De hecho se dio el caso de una sola persona, que se identificaba como vasca, que categorizó a amigos de la misma nacionalidad institucional, española, como extranjeros.
} 


\section{Diferencias sistemáticas}

En la sección anterior, puramente descriptiva, hemos asumido, de acuerdo con nuestra hipótesis, que la percepción de los amigos transnacionales en tanto que "no extranjeros" es debida al propio establecimiento relaciones de amistad y a la calidad de ésta. Sin embargo, es evidente que esto puede ser debido a otros factores - por ejemplo, ideológicos, cognitivos o en el peor de los casos a aspectos metodológicos ${ }^{15}$ - y sencillamente reflejarse en ciertas relaciones. En esta sección estudiamos las diferencias sistemáticas entre las relaciones a las que se aplican categorizaciones distintas tanto para aprender más a propósito de los efectos de la amistad como para situar los efectos de las dimensiones ideológicas e institucionales.

Antes de comenzar, especifiquemos cuatro elementos en tres niveles analíticos a examinar para estudiar tales diferencias sistemáticas: 1) Nivel de los individuos, egos y alteri; 2) nivel de las relaciones: díadas; y 3) nivel de la red personal: tríadas y zona de primer orden. En efecto, las diferencias en la designación de ciertos amigos transnacionales en tanto que no extranjeros pueden ser debidas a: 1) las características de los egos que responden. En efecto, ciertas personas pueden ser, por distintas razones, más "abiertas", más inclusivas, o más permeables en sus cogniciones que otros. 2) También pueden ser debidas a diferencias sistemáticas entre los alter. Es posible imaginar que ciertas personas con características específicas sean más fácilmente considerables como "noextranjeros". 3) También, de acuerdo con nuestra hipótesis, la propia dinámica de las relaciones de amistad puede hacer que, dada la calidad de la relación, ciertos alter sean menos percibidos como extranjeros. 4) Finalmente, la posición de la relación transnacional en la red personal de ego puede hacer que ciertas relaciones, las más inmersas en mi opinión, sean menos percibidas como extranjeras.

Apoyándonos en estos distintos niveles de análisis, queremos examinar la incidencia de aspectos institucionales, ideológicos y relacionales en la caracterización de ciertos alteri en tanto que extranjeros.

\section{1) Hipótesis a nivel macro}

1.1 Las instituciones tienen el poder de definir quién pertenece y quién no en términos legales de ciudadanía. La percepción de ciertos alter en tanto que extranjeros puede ser influida por los términos institucionales. En este caso,

\footnotetext{
15 Hemos realizados análisis tanto sobre el protocolo, como sobre aspectos cognitivos. No los presentamos aquí por falta de espacio.
} 
podríamos imaginar que, dado que existe una semi-ciudadanía o ciudadanía secundaria Europea, los alteri ciudadanos de estados miembros de la UE, o europeos en general, no fueran considerados como extranjeros.

1.2 Los modelos ideológicos de la nación tienen tradiciones diferentes. Algunos estados tienen tradiciones basadas en ius soli, la civilización y la ciudadanía (Francia), otras tienen tradiciones más etno-culturales basadas en ius sangui, la cultura y la nacionalidad (Alemania). Algunos estados tienen una tradición de definición universalista, abstracta y directa de la ciudadanía (Francia de nuevo), en otras la ciudadanía es mediada por la pertenencia intermedia a comunidades o "regiones históricas" (Gran Bretaña o España). Además de los modelos ideológicos de los estados (en los que se socializaron los individuos), los individuos también pueden adherirse más o menos a ellos, según sus ideologías de identificación y pertenencia. Los modelos ideológicos, tanto si son heredados de las tradiciones nacionales, como individuales, probablemente afecten a cómo se considere a los alteri en tanto que extranjeros o no.

\section{2) Hipótesis a nivel meso}

2.1 Amistad, confianza y solidaridad diádica. Conforme la confianza y la solidaridad se va estableciendo entre los amigos, conforme se van convirtiendo en iguales, tal como lo postula la amistad entre ellos, pero también en más similares debido al ajuste recíproco del comportamiento y al establecimiento de nuevas normas comunes (Por ejemplo, para que dos amigos puedan comer juntos es necesario que se pongan de acuerdo en cuál es la definición de la "comida": ¿se trata de alimentos calientes o fríos?, ¿en gran cantidad o tan solo un poco de pan con queso o embutido?, ¿a las 12:00 o a las 15:00? etc...) se considerarán cada vez, evidentemente como menos extraños, y por lo tanto menos como extranjeros. Conforme su amistad y confianza se hacen más profundas (por ejemplo, al haber sido demostradas en el tiempo o mediante contenidos específicos de la relación) y la relación de amistad puede soportar la descontextualización y conducir a un acercamiento de la díada a la esfera más privada (Allan 79), las otras características sociales, por lo tanto la nacionalidad, ya no son relevantes, y por lo tanto son olvidadas o pasan desapercibidas.

2.2 La red como garantía de la confianza y la solidaridad. La confianza interpersonal diádica también puede ser sustituida y/o reforzada por la inscripción de la relación en la red personal: cuando alteri importantes en los que ya se tiene confianza, consideran a un tercer alter como digno de confianza y receptor de solidaridad, es más probable que ego lo haga también. Cuando el comportamiento de los alteri 
significativos para ego sugieren que el alter transnacional es digno de confianza y solidaridad y/o forma parte del grupo, es más probable que ego no considere a alter como extranjero.

Para probar estas hipótesis, examinamos la incidencia de diversas variables en la percepción de un alter transnacional en tanto que extranjero. Presentamos un sumario de los resultados de las variables por nivel de análisis en la siguiente tabla ${ }^{16}$.

Tabla 2. Sumario de los resultados*.

\begin{tabular}{|c|c|c|}
\hline Nivel & Variable & Significatividad \\
\hline Ego & Nacionalidad & $\mathrm{Si}$ \\
\hline Ego & Identificaciones principales & $\mathrm{Si}$ \\
\hline Ego & Actitudes Europeas & $\mathrm{Si}$ \\
\hline Alter & Ciudadano UE o Europeo & $\mathrm{No}$ \\
\hline Díada & Fuerza del lazo & $\mathrm{Si}$ \\
\hline Red & Imbricación del lazo & $\mathrm{Si}$ \\
\hline Red & Densidad de la red & $\mathrm{Si}$ \\
\hline
\end{tabular}

(*) Para más detalles sobre las relaciones entre variables ver las tablas 4, 5 y 6 en el anexo.

Comentamos a continuación de manera más detallada los resultados hallados en los análisis en relación con las hipótesis en ambos niveles macro y meso.

\section{Resultados del nivel macro}

1.1 Instituciones: al existir una semi-ciudadanía o ciudadanía secundaria Europea instituida y garantizada por la UE, los alteri ciudadanos estrictos de la UE o, por extensión, todos aquellos europeos, serían menos percibidos como extranjeros. En esta hipótesis tuvimos una gran sorpresa: ni los ciudadanos de la UE, ni los europeos en general son menos frecuentemente percibidos como extranjeros que los no europeos. La UE no tiene la capacidad directa de incidir en la percepción de extranjería de los alteri. Si esto ocurre finalmente, debe ser gracias a caminos indirectos que pasan por el nivel meso, el nivel micro o ambos.

1.2 Ideologías. Los modelos ideológicos, más o menos inclusivos, de la nación y la pertenencia deben afectar a la manera en que los alteri son percibidos o no en

\footnotetext{
16 La tabla y los comentarios presentados están basados en análisis que utilizan - por ahora- métodos estadísticos sencillos: Chi-cuadrado para probar la dependencia entre variables, fi y gamma de Goodman y Kruskall para determinar la intensidad de la dependencia. Prevemos en un futuro cercano, realizar análisis de regresión para controlar las interdependencias entre variables y presentar un modelo en que sólo aparezcan los efectos significativos. Pensamos utilizar regresión multinivel, dado que las diadas no son independientes, sino que están ligadas al ego respondente. Esto permitirá distinguir la importancia de distintas variables actuando al nivel individual, diádico y de la red.
} 
tanto que extranjeros. Aquí podemos distinguir los modelos ideológicos en tanto que estos pueden manifestarse según la tradición nacional de los países de los egos, cuya variable sería la nacionalidad, pero también, según los modelos de identificación propios a los egos.

En cuanto a las tradiciones nacionales por países, encontramos (teniendo en cuenta sólo las nacionalidades más representadas en la muestra) que ciertas nacionalidades se muestran más inclusivas y abiertas al considerar menos frecuentemente a los alteri en tanto que extranjeros y ciertas otras menos inclusivas. La media de no considerar a un amigo transnacional como extranjero es del $82 \%$, los franceses (92\%), italianos (90\%) y alemanes (89\%) se muestran más inclusivos, mientras que los españoles $(80 \%)$, pero especialmente los británicos $(62 \%)$ resultan más cerrados. Aunque la muestra es pequeña para sacar grandes conclusiones, parecería que las naciones que se apoyan en pertenencias a comunidades o regiones históricas como mediadoras de la nacionalidad son menos inclusivas que aquellas que se basan en valores universalistas de ciudadanía y, ya sea la civilización o la cultura.

Para reconstruir el sistema de identificación de los egos les pedimos que dijeran en qué medida se sentían identificados, en una escala de cuatro puntos (nada, un poco, bastante, mucho) con diferentes tipos de comunidades y contextos (nación, cultura, idioma, tendencia política, movimientos sociales, profesión, religión, familia y amigos).

El análisis factorial de estas variables (Figura 3) muestra un espacio cuyos dos factores principales están estructurados, el primero, por la identificación en general, y en particular por los elementos nacionales (nación, cultura y lengua). El segundo factor, positivamente por elementos políticos (ideas políticas y movimientos sociales) y negativamente por elementos tradicionales (religión, familia y trabajo). Los egos con puntuaciones altas en el polo "político", tienden a considerar menos como extranjeros a sus amigos transnacionales. Por el contrario, los egos con valores altos en el polo "tradicional" tienden a considerarlos algo más a menudo como extranjeros. El polo "nacional" sin embargo, no parece tener una influencia clara. 
Figura 3. Análisis factorial de identificaciones y extranjería

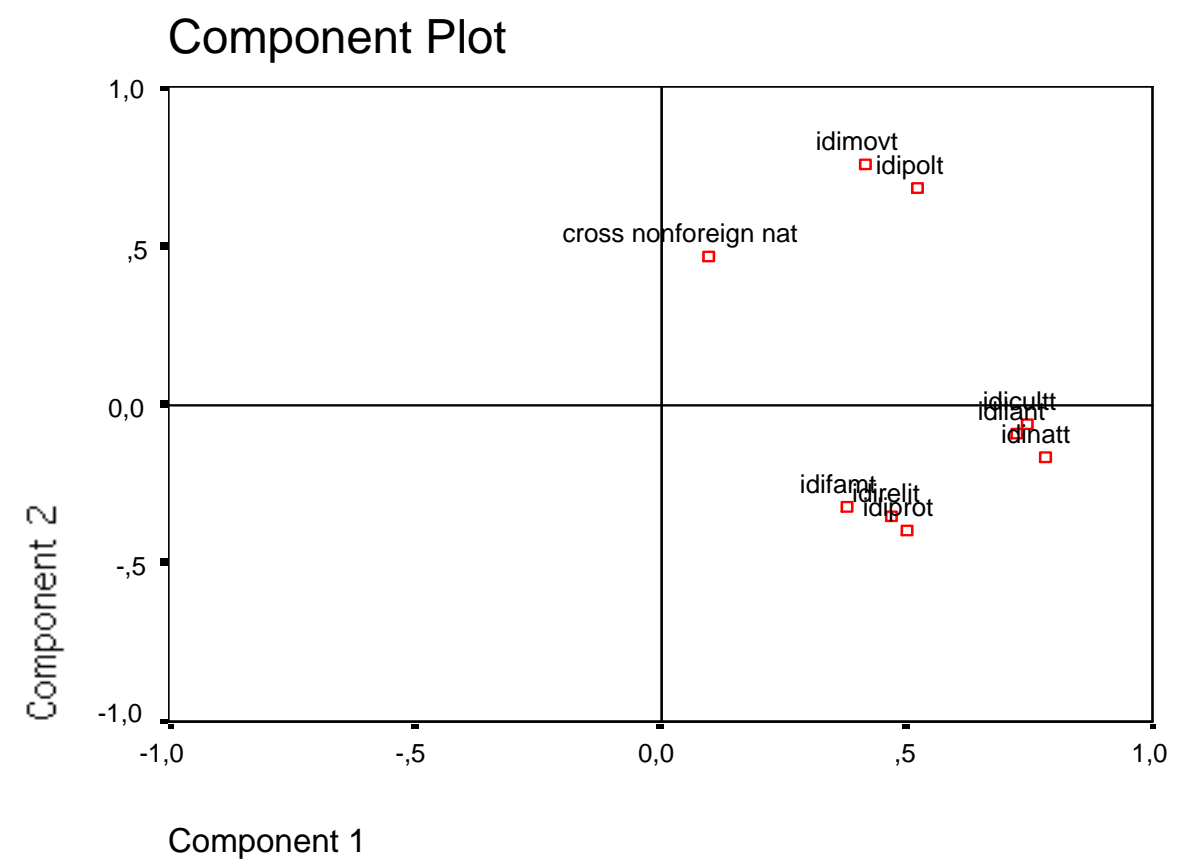

*Los dos factores explican el $49,6 \%$ de la varianza.

Estos resultados podrían encajar con los hallazgos de Duchesne y Frognier (1995) quienes afirman que la identidad nacional no es contradictoria o competidora con la identidad Europea, sino que por el contrario, aquellos individuos más socializados ya en un ideal de nación abstracto y universalista son más proclives a aceptar la identidad Europea.

En cuanto a las actitudes Europeas de los egos, en general, aquellos con actitudes Europeas, medidas a partir de preguntas estándar de los Eurobarómetros, tienden a considerar menos frecuentemente como extranjeros a los alteri transnacionales ${ }^{17}$.

\section{Resultados del nivel meso}

A nivel meso, en que proponemos los aspectos más originales de nuestra investigación, encontramos resultados muy interesantes. También es en este punto donde los resultados son al mismo tiempo más coherentes y más matizados.

\footnotetext{
17 En este punto no es posible identificar cuál es la causa y cual la consecuencia: ¿Son las actitudes europeas las que provocan que las amistades no sean consideradas extranjeras o, es que cuando ego, gracias a la formación de la amistad, tiene suficientes amigos transnacionales que no considera extranjeros, aumentan sus actitudes europeas? Por ahora sólo podemos constatar la coocurrencia de ambas e intuir que la causalidad es en ambos sentidos.
} 
2.1 Amistad, confianza y solidaridad diádica. Conforme la confianza y la solidaridad se va estableciendo entre los amigos y estos se van convirtiendo en similares y en iguales, los amigos deberían tender a no considerarse extranjeros. Conforme la amistad es profundizada, la nacionalidad debería llegar incluso a ser olvidada.

Esta hipótesis se vio fuertemente confirmada a partir de todos los indicadores considerados. En efecto, todas las medidas sobre la fuerza de la díada indican que el establecimiento de una relación de confianza y solidaridad producen que los alteri transnacionales no sean considerados extranjeros. Encontramos diferencias sistemáticas muy interesantes, no sólo entre los alteri considerados como extranjeros o no, sino también entre los alteri no considerados extranjeros cuya nacionalidad es percibida y aquellos cuya nacionalidad es olvidada o pasa desapercibida.

2.1.1 Relaciones en que los alteri no son considerados extranjeros. Estas relaciones son más fuertes que aquellas en que los alteri con considerados extranjeros. Parece que para no considerar a un alteri transnacional como extranjero, es requerida cierta fuerza de la relación, pero no mucha: que alter sea un "conocido" no es suficiente, pero una relación amistosa basta. Sin embargo, es necesaria cierta cantidad de interacción voluntaria (por teléfono o e-mail), en cambio la interacción frecuente cara a cara, probablemente más dependiente de contextos de actividad, no es suficiente. Sin embargo, la relación no requiere ser muy larga, encontramos el umbral máximo en un año de relación. Los intercambios de pistas y consejos generales, hablar del futuro, de Europa, o de viajes, son contenidos de conversación relacionados con no considerar a alter como extranjero. Otros contenidos más comprometidos, como prestar cantidades de dinero importantes para un estudiante (150€), estar dispuesto a cuidar en caso de enfermedad, o hablar de cuestiones amorosas y problemas personales, también influyen en que alter no sea considerado extranjero, sin embargo la puntuación más alta es para un contenido relativamente "ligero": sencillamente salir a pasarlo bien.

2.1.2. Relaciones en que además se olvida la nacionalidad de alter. Para olvidar la nacionalidad de alter es requerida una relación mucho más consolidada. Una 'relación amistosa' puede ser suficiente, pero es mejor que se trate de un 'amigo', aunque no es necesario que se trate de un 'mejor amigo'. La duración de la relación requiere ser mayor: 2 o 3 años son necesarios por lo menos, y el umbral máximo es a los 5 años de relación. Por otro lado, estas relaciones soportan bien un contacto cara a cara poco frecuente (menos de una vez al mes), dado que a menudo estos alter viven más lejos de $500 \mathrm{~km}$ de ego. En cuanto a los contenidos se refiere, hablar del futuro, prestar dinero (150€), estar dispuesto a invertir $3800 €$ 
en un negocio con alter, pero especialmente hablar de cuestiones amorosas y problemas personales, o estar dispuesto a cuidar en caso de enfermedad, son los contenidos más relacionados con olvidar la nacionalidad de alter. También un cierto grado de multiplicidad de las relaciones está asociado con olvidar la nacionalidad de alter. Todo esto muestra que las relaciones en que es posible olvidar la nacionalidad requieren mayores niveles de confianza y solicitud.

Vemos claramente a este nivel cómo la amistad tiene un impacto en definir la "pertenencia" designando las fronteras de la extranjería ya al nivel diádico, es decir la mera existencia de lazos entre pares de actores. Hemos visto que, incluso más lejos, que cuando se llega a niveles de confianza y solidaridad altos entre los pares de actores, la cuestión de la nacionalidad puede incluso desaparecer del campo de relevancia de la relación: cuando la confianza y la solidaridad existe en la esfera privada, la solidaridad abstracta apoyada por la nacionalidad se vuelve innecesaria, por lo tanto olvidada.

\subsection{Redes como garantía de la confianza y solidaridad.}

Más allá del nivel diádico, queremos examinar cómo la imbricación (o no) de una relación en una red tiene un efecto adicional para que alter sea considerado o no como extranjero. En efecto, la relación entre alter y otros alteri importantes para ego pueden favorecer una percepción más inclusiva de alter. Cuanto menos extraño es alter, menos extranjero es, esto ya aparece en el nivel diádico, tal vez del mismo modo cuando un alter es menos considerado como extraño por los otros alteri del entorno de ego, este tiende a no considerarlo como extranjero. En este caso, los alteri están funcionando como una garantía de la pertenencia de alter al mundo micro social de ego, lo que también tiene un impacto en la percepción de ego de la pertenencia de alter a su mundo macro-social (su extranjería).

Tenemos dos indicadores de la imbricación de alter en la red. Nuestra primera medida es un indicador estándar de relación entre los alteri: preguntamos a ego si cada uno de los alter conocía a los cinco mejores amigos de ego. Además, más allá del simple conocimiento, preguntamos a ego cómo percibía la calidad de la relación entre los alteri y sus cinco mejores amigos. Las respuestas ofrecidas eran: 'conflicto', 'desconocido', 'conocido', 'relación amistosa', 'amigos', 'mejores amigos'. Esto permite ver en qué medida la simple imbricación (simplemente conocerse) puede explicar las variaciones en las fronteras de extranjería, o si un cierto grado de confianza y solidaridad entre los alteri es necesario como garantía de la pertenencia y no extranjería de alter. 
Los datos mostraron que el simple conocimiento entre alteri no tiene ningún papel. El hecho de que un alter transnacional conozca o no a los mejores amigos de ego no hace que sea menos percibido como extranjero. Sin embargo, cuando el alter transnacional tiene un cierto número de 'relaciones amistosas', o incluso mejor de 'amigos' entre los mejores amigos de ego, es más probable que no sea considerado como extranjero. Nuestro análisis muestra que el máximo es tener al menos 3 'relaciones amistosas' o al menos 2 'amigos' entre los mejores amigos de ego para no ser considerado extranjero. Mayores niveles de confianza entre los alteri parecen requerir menos relaciones adicionales para garantizar la pertenencia de alter al mundo micro-social de ego.

De modo similar, examinamos cómo la imbricación influye en que ego, no sólo no considere a alter como extranjero, sino que olvide su nacionalidad. Hemos visto en la sección anterior que las relaciones en que ego olvida la nacionalidad de alter tienden a ser más consolidadas, más largas y más fuertes. Si el argumento adelantado en el párrafo anterior es cierto (mayores niveles de confianza entre alteri requieren menos relaciones adicionales para garantizar la pertenencia de alter al mundo micro-social), entonces estas relaciones deberían requerir imbricación en menos relaciones para garantizar la confianza o imbricación en relaciones menos fuertes, ya que la propia díada es portadora de mucha confianza. Esto es en efecto lo que hallamos: aunque la probabilidad de que la nacionalidad de alter sea olvidada crece constantemente conforme está imbricado en 'relaciones amistosas', esta dependencia, aunque significativa, es debil (claramente mucho menos fuerte que el efecto de la imbricación simplemente para no considerar a alter como extranjero). Por otro lado, la amistad entre alter y los mejores amigos de ego es apenas significativa. Así pues, el proceso mediante el que olvidamos la nacionalidad de un alter transnacional parece más ligado a un proceso diádico de amistad y al establecimiento de confianza más privada e interpersonal.

Nuestra segunda medida de imbricación consistió en preguntar a ego (entre otros contextos habituales de interacción) si a menudo veía a alter en un grupo de amigos, y si lo veía a menudo en solitario. Esta pregunta combina la imbricación interpersonal (para verse con otros amigos es necesario conocerse) con el contexto particular habitual de verse con alter: un alter encontrado a menudo con un grupo de amigos, dado que la noción de 'grupo' incluye la percepción de una pertenencia común, puede ser más fácilmente identificado como alguien que 'pertenece' al mundo micro-social y por lo tanto tal vez más fácilmente percibido como no extranjero. Los datos muestran que la interacción habitual con alter en un grupo de amigos tiene impacto en que alter no sea considerado extranjero, sin embargo, no 
tiene ningún efecto, de acuerdo con los resultados anteriores, en que la nacionalidad de alter sea olvidada. Por otra parte, encontrarse a solas con alter no tiene efectos de ningún tipo.

Por último, las redes más densas, es decir, en que los amigos se conocen más y/o son más amigos entre sí, independientemente de si los lazos son transnacionales o no, permiten que aquellos alter transnacionales sean incluidos más fácilmente y menos considerados como extranjeros. Las redes más densas y amistosas son más inclusivas con los alteri transnacionales.

Así pues, vemos que tanto las díadas como las redes de amistad inciden en la delimitación de las fronteras de extranjería en el ámbito privado.

\section{Conclusiones y desarrollo futuro}

En este artículo hemos pretendido explicar la identificación de los individuos, a partir de procesos macro y meso, aplicado a la identidad europea en el contexto actual de integración institucional. Hemos distinguido entre la identificación en la esfera privada y la identificación en la esfera pública. Hemos operacionalizado la identificación en la esfera privada a partir de la categorización de los alteri en tanto que extranjeros o no. La identificación en la esfera pública haría referencia a las identidades proclamadas en los discursos de los individuos. En este artículo sólo hemos examinado las identidades en la esfera privada.

Los resultados hallados, volviendo al esquema del "barco" de Coleman (1992) sobre las relaciones micro-macro, podrían ser expresados de esta manera: 


\section{Nivel macro}

2) Discursos ideológicos

1) Ciudadanía instituciona〉

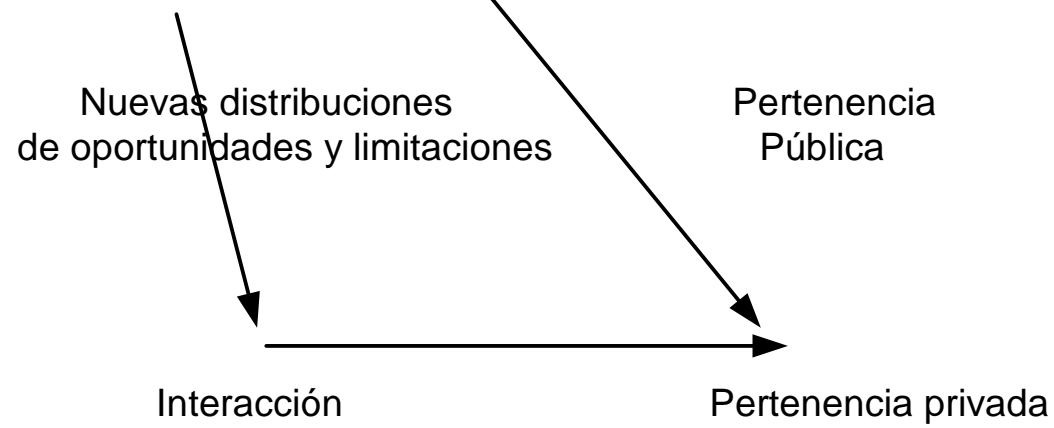

3) Procesos de amistad: selección, influencia, regulación diádica y triádica, establecimiento de igualdad, confianza y solidaridad.

\section{Nivel micro}

1. El establecimiento de la semi-ciudadanía o ciudadanía secundaria Europea garantizada por la Unión Europea favorece las posibilidades para la interacción europea y por lo tanto la emergencia de nichos y estilos de vida "europeos". Sin embargo, la definición institucional de ciudadanía europea no produce directamente alteraciones en las fronteras de la extranjería al nivel privado, si esto ocurre finalmente, entonces debe ser gracias a caminos indirectos que pasen por el nivel meso o el nivel micro.

2) Los discursos ideológicos sobre la nacionalidad, la pertenencia, la identificación, tanto si son heredados de las tradiciones nacionales por la socialización de los egos como si corresponden a sus propios sistemas de identificación, muestran efectos en las fronteras de identificación al nivel privado.

3) El establecimiento de relaciones de amistad y la imbricación en redes de amistad tienen un efecto muy claro en las fronteras de identificación al nivel privado. Conforme se van estableciendo relaciones de confianza y solidaridad, tanto si la garantía de estas reposa en el nivel diádico como en la red, las fronteras cognitivas entre ego y alter tienden a atenuarse, incluso a desaparecer cuando la confianza ya es muy consistente.

El siguiente paso lógico de nuestra investigación sería examinar cómo los factores macro y meso inciden en las pertenencias pública, esto es las identidades aceptadas y proclamadas por los individuos. 


\section{Figura 5. (Continuación)}

\section{Nivel macro}

2) Discursos ideológicos $-\cdots \cdot-\cdot \cdot \cdots \cdot \cdots \rightarrow$ dentidad europea

1) Ciudadanía instituciona $-\cdot-\cdot-\cdot \bar{i}$

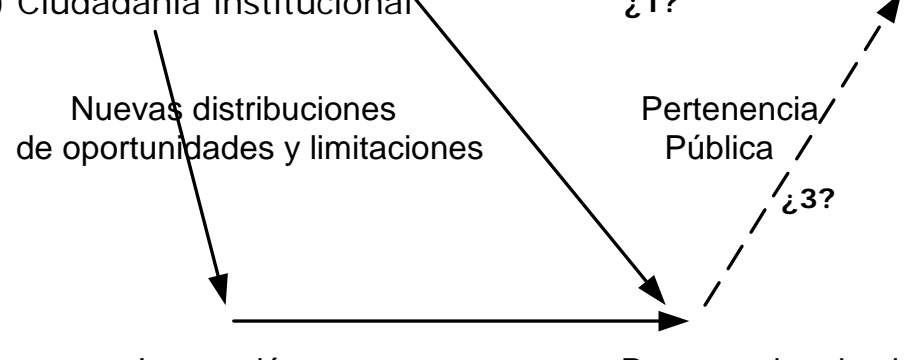

Interacción

Pertenencia privada

3) Procesos de amistad: selección, influencia, regulación diádica y triádica, establecimiento de igualdad, confianza y solidaridad.

\section{Nivel micro}

Nuestras hipótesis respecto a estos niveles son las siguientes:

1) La ciudadanía institucional europea no ha sido capaz de tener un efecto a nivel privado en la identificación de los individuos. Por lo tanto suponemos que, de igual modo, hoy en día tampoco tenga una especial incidencia en la identificación pública. No esperamos que los ciudadanos de la UE se consideren como europeos más frecuentemente, o con mayor intensidad que los demás europeos.

2) Los discursos sobre la identidad heredados de las tradiciones nacionales y los sistemas identitarios personales sí han tenido un efecto en las identificaciones privadas. Esperamos, dado que se trata de la misma naturaleza de fenómenos, y que estos parecen comportarse de forma sistémica, que estos discursos tengan un papel tanto más importante en la aceptación y adopción de la identidad europea.

3) El movimiento de las fronteras de extranjería en el ámbito privado aparece más claramente producido por la amistad y los procesos de redes mediante la creación de similitud, igualdad, solidaridad y confianza entre los amigos. Sin embargo, para que la amistad, considerada en nuestras sociedades como una relación relegada a la esfera privada, tenga una incidencia que trascienda a la esfera pública probablemente sea necesario algo más. Proponemos dos posibles mecanismos compatibles entre sí:

3.1) Efectos propios a las redes: La presencia de relaciones numerosas con europeos (aunque a priori estos no sean considerados como menos extranjeros), especialmente si éstas son fuertes tanto en términos diádicos como en términos 
estructurales, y tal vez según las características generales de la red personal, puedan, mediante procesos de requilibrio cognitivo, favorecer la emergencia, o aumentar la importancia relativa, de los sentimientos de pertenencia e identificación con comunidades imaginadas capaces de incluir a los miembros de las comunidades personales sin que estas estén determinadas a priori.

3.2) Por la mera existencia de un marco legal de ciudadanía europea, el resultado de producción de igualdad e identidad en los procesos meso de amistad puede ser justificado a posteriori en beneficio de la identifiación europea, tanto si se trata de un proceso consciente como si no. Así, aunque la semi-ciudadanía garantizada por la UE no sea capaz de producir adhesion a la identidad europea, al crear la categoría institucional de "llegada" (además de facilitar la interacción), puede permitir la justificación a posteriori de un proceso que, en realidad, es efectivamente activado y producido por procesos meso y micro. Si esto es así, entonces se cierra el ciclo macro-micro-macro.

Esta es una cuestión abierta por el momento pero, si fuera confirmada, la UE tendría interés en reforzar los efectos de la lógica de la amistad, dada la posibilidad de "recuperar" al nivel macro las solidaridades aparecidas a nivel micro, ya que estas aparecen "certificadas" o garantizadas por la ciudadanía secundaria o semiciudadanía europea.

\section{Bibliografía}

Allan, G. H. (1979). A sociology of friendship and kinship. London: George Allen and Unwin.

Anderson, B. (1983). Imagined communities: reflections on the origin and spread of Nationalism Verso [1991].

Coleman, J. S. Farraro, T. J. (1992). Rational choice theory: Advocacy and critique. London: Sage Publications.

Duchesne, S. and Frognier, A-P. (1995). "Is there a European Identity", en O. Niedermayer and R. Sinnott (eds) Public Opinion and Internationalized Governance. New York: Oxford University Press, p 193-226.

Eisenstadt S.N. (1956). From generation to generation, age groups and social structure. Free Press: Glencloe.

Eisenstadt S.N. Roninger (1984). Patrons, clients and friends. Cambridge: Cambridge University Press. 
de Federico, A. (2001). "Networks and identities: Borders of solidarity and borders of identification of European exchange students". Presentado en 'Networks And Transformations' organised by the Global Studies Association (GSA), Manchester Metropolitan University, Manchester, UK, 2-4 julio. <http://www.mmu.ac.uk/gsa/first_conference.html>

Ferrand A. (1993). L'Analyse des réseaux personnels. Habilitation à diriger des recherches en sciences sociales et humaines auprès de I'Université de Lille. En especial las páginas XXXIX a LVIII.

Fichte, Johann Gottlieb (1988). Discursos a la nacion alemana. Madrid: Tecnos, 1988.

Habermas, J. (1999). La inclusión del otro. Barcelona: Paidós.

Hansen, P. (2000). "'European Citizenship', or where neoliberalism meets ethnoculturalism. Analyzing the European Union's citizenship discourse » European Societies, 2(2): 139-165.

Lamo de Espinosa, E. (Ed.) (1995). Culturas, Estados, Ciudadanos: Una aproximación al multiculturalismo en Europa. Madrid: Alianza Editorial.

Manitakis, N (2000). "Les migrations estudiantines en Europe, 1890-1930" en Leboutte, R (ed), Migrations et migrants dans une perspective historique. Permanences et innovations / Migrations and migrants in Historical Perspective. 1997-8, EUI, Florence, October 1997, Bruxelles. P.I.E. -Peter Lang (Series “Multiple Europe" № 12), 346 p, (agotado).

Martineau, M. (1995). Regard étudiant sur les échanges communautaires et cinéma en Europe. INRP. Cinéma Action, 4o trim. № 77.

Mendras, H. (1997). L'Europe des Européens. Paris: Ed. Gallimard.

Paine, R. (1969). "In search of friendship", Man, vol. 4.

Pomian, K. (1990). L'Europe et ses Nations. París: Gallimard.

Ruiz-Gelices, E., King, R. Favell, A. (2000). “International Student Migration in Europe and the Institutionalisation of a 'European Identity'" International Migration: New Patterns, New Theories, 11-13 September, Nottingham Trent University Schnapper, D. (1994). La communauté des citoyens: sur l'idée moderne de Nation. París: Gallimard. 
Soysal, Y. (2001). Plenary session at the 5th Conference of the European Sociological Association "Visions and Divisions. Challenges to European Sociology", Helsinki 28 de agosto - 1 de septiembre.

Strath, Bo, Triandafyllidou, Anna. EURONAT, Representations of Europe and the nation in current and prospective member-states:media, elites and civil society. Proyecto financiado por el V Programa Marco de la UE. Investigación en curso.

Therborn, G. (1999). "'Europe' as issues of sociology" in T. P. Boje, B. van Steenbergen, B. Walby, S. European Societies: Fussion or Fission? London: Routledge.

Touraine, A. (1999). «European sociologists between economic globalization and cultural fragmentation » in T. P. Boje, B. van Steenbergen, B. Walby, S. European Societies: Fussion or Fission? London: Routledge.

\section{Anexo metodológico}

